\title{
Aplicación de la giberelina (Ryz up), para inducir la brotación en tubérculos de la papa (Solanum
} tuberosum) variedad canchán INIA

\section{Application of gibberellin (Ryz up), to induce sprouting in tubers of the potato (Solanum tuberosum) canchán INIA variety}

Eyner Huamán Huamán ${ }^{1 *}$, Lily del Pilar Juárez Contreras ${ }^{1}$, Juan Carlos Neri Chavez y Roicer Collazos Silva

\section{RESUMEN}

En el presente estudio se evaluó el efecto de tres dosis de la giberelina Ryzup sumergidos en dos tiempos diferentes para inducir la brotación en papa (Solanum tuberosum) variedad canchán-INIA. Se utilizó un diseño completamente aleatorizado con seis tratamientos más un testigo absoluto. Las dosis de aplicación fueron 2,5, 5 y 7,5ml por 201 de agua. Los tiempos de inmersión presentaron dos niveles, de 5 y 15 minutos, respectivamente. Posteriormente utilizando el software libre R se efectuó el análisis de varianza y la prueba de comparaciones múltiples según Duncan por tratamientos. Las variables evaluadas fueron: porcentaje de la brotación, número de brotes por tubérculo, longitud de brotes por tubérculo y diámetro de brotes por tubérculo. Los resultados a los 33 días de haber instalado el ensayo mostraron que el T7 (7,5ml de Ryzup sumergidos durante 15 minutos) obtuvo los valores más altos y uniformes, exhibiendo un porcentaje de brotación del 85,26\%,8,36 brotes por tubérculo, 7,61 cm de longitud, y un diámetro de 5,41 cm por tubérculos. Se concluye que la aplicación de giberelinas acelera el proceso de brotación e induce el rompimiento del estado de reposo de las semillas, lo cual queda demostrado en el T7 que logró obtener los mejores valores a los 33 días, lo que resulta útil y accesible al agricultor ya que se traduce en una ganancia de tiempo de hasta dos meses, y posibilita la realización de hasta tres ciclos del cultivo al año.

Palabras clave: Fitohormonas, dormancia, ácido giberelico.

\begin{abstract}
In the present study we evaluated the effect of three doses of gibberellin Ryzup submerged in two different times to induce sprouting in potato (Solanum tuberosum) canchán-INIA variety. A completely randomized design with six treatments plus an absolute control was used. The application rates were $2.5,5$ and $7.5 \mathrm{ml}$ per 201 of water. The immersion times had two levels, 5 and 15 minutes, respectively. Subsequently using the free software R was performed the analysis of variance and the test of multiple comparisons according to Duncan by treatments. The variables evaluated were: percentage of sprouting, number of shoots per tuber, length of shoots per tuber and diameter of shoots per tuber. The results 33 days after the test were installed showed that T7 (7.5ml of Ryzup submerged for 15 minutes) showed the highest and uniform values, exhibiting $85.26 \%$ sprouting percentage, 8.36 sprouts per tuber, $7.61 \mathrm{~cm}$ in length, and a diameter of $5.41 \mathrm{~cm}$ per tubers. It is concluded that the application of gibberellins accelerates the budding process and induces the breaking of the resting state of the seeds, which is demonstrated in the T7 that managed to obtain the best values at 33 days, which is useful and accessible to the farmer cause it translates into a gain of time of two months, and allows the implementation of three crop cycles per year.
\end{abstract}

Keywords: Phytohormones, dormancy, gibberellic acid.

\footnotetext{
Universidad Nacional Toribio Rodríguez de Mendoza, Chachapoyas, Amazonas, Perú
}

*Autor de correspondencia. E-mail: eynerhuaman@indes-ces.edu.pe 


\section{INTRODUCCIÓN}

La papa ha sido y sigue siendo uno de los alimentos más importantes en la lucha contra el hambre y la pobreza en el Perú y muchas partes del mundo, resultando ser el tubérculo (Fernie y Willmitzer, 2001; Cerna, 2011) y el tercer alimento agrícola más consumido en el mundo después del arroz y el trigo (Hancock et al., 2014).

En lo que respecta a nivel mundial, China ocupa el primer lugar en la producción de papa con 89 millones de toneladas, seguido por la India, Rusia, Ucrania, Estados Unidos, Alemania, y Francia, respectivamente (MINAGRI, 2015), juntando a nivel mundial totales de hasta 300 millones de toneladas de producción (Birchetal., 2012).

Por otra parte el Perú lidera la producción de papa en Latinoamérica y es el décimo cuarto mayor productor a nivel mundial. La producción de papa en Perú alcanzó los 4,6 millones de toneladas en 2014, en una superficie sembrada de 317648 hectáreas, con un rendimiento promedio de 14,7 th/ha (MINAGRI, 2015). Además, el cultivo de papa representa el 3\% del PBI agrícola del Perú, mayor que cualquier otro cultivo alimenticio nacional y es trabajado actualmente por más de 111000 productores. Los tubérculos de papa son ricos en varios micronutrientes y vitaminas $\mathrm{C}$ y en muchos países desarrollados representan un cultivo básico (Senés-Guerrero et al., 2013). En la región de estudio, los Andes, la papa constituye el principal cultivo básico, con un promedio por familia de 10 a 12 cultivares, de aproximadamente 4300 variedades nativas de papa andina (Brush et al., 1995). Asimismo, el consumo per cápita en nuestro país se ha incremento, de 73 kilos por persona al año en el 2007, pasamos a consumir 86 kilos el 2014 (MINAGRI, 2015).

E1 40\% de las papas sembradas en Perú son de la variedad INIA 303 - Canchán, lo que significa aproximadamente 120000 hectáreas cada año, con un rendimiento a nivel de productores de hasta $30 \mathrm{tn} / \mathrm{ha}$ (INIA, 2012). La papa se propaga vegetativamente por medio de los tubérculos (a los que se les da el nombre genérico de semilla), lo cual permite mantener su constitución genética inalterable (Peña, 2015).

Generalmente se consideran dos factores estrechamente ligados a la emisión de brotes de las semillas de papa: la variedad de la papa, que es la responsable de la duración del período de reposo y la edad fisiológica del tubérculo semilla, la cual depende de las condiciones de almacenamiento a las cuales ha sido sometida la semilla (Peña, 2015). La temperatura también es uno de los factores más importantes que afectan a la papa tanto en su crecimiento como en su cultivo (Levy y Veilleux, 2007). Fisiológicamente el tubérculo semilla sufre cambios desde la cosecha hasta cuando muestra brotes múltiples y vigorosos, dentro de ellos el estado de reposo o dormacia es el más resaltante (Torres et al., 2011). La dormancia de un tubérculo de papa (Solanum tuberosum) se define como el estado fisiológico en el que no se producirá crecimiento autónomo del brote, incluso cuando el tubérculo se coloca en condiciones ideales para el crecimiento del brote (Reust, 1986). La inactividad comienza en el momento del inicio del tubérculo y su duración se ve influenciada por la edad del tubérculo y las condiciones ambientales que prevalecen durante el desarrollo del tubérculo en la planta madre y después de la cosecha (Vreugdenhil, 2007). El final del periodo de reposo de una semilla de papa está relacionado a un balance asociado con una disminución de sustancias inhibidoras y un aumento de sustancias promotoras de crecimiento (Vejarano y Morales, 2014). Es así que culmina cuando el tubérculo ha desarrollado uno o más brotes de por lo menos $3 \mathrm{~mm}$ de largo, que generalmente depende de: (i) la variedad; (ii) el estado de maduración en el momento de la cosecha; (iii) la temperatura durante la época de crecimiento vegetativo; (iv) el tamaño del tubérculo-semilla; (v) las condiciones de almacenamiento (luz, temperatura y humedad); y (vi) los daños causados al tubérculo, que normalmente se mantienen entre tres a cuatro meses. Existen diferentes métodos de tratamiento de ruptura del período de reposo, mediante tratamientos térmicos, corte del tubérculo y fitohormonales (Torres et al., 2011). Diferentes autores mencionan que fitohormonas como la giberelina 
aceleran el proceso de germinación y brotación, y son importantes para inducir el rompimiento de la latencia de semillas (Deaquiz y Burgos, 2013). Por otro lado, aplicaciones de giberelinas, citoquininas y auxinas también rompen el reposo de los tubérculos. Además la aplicación de giberelinas provoca la acumulación de reguladores en niveles adecuados para permitir el proceso de división y agrandamiento celular que conduce al rompimiento de la dormancia e induce a la aparición temprana de brotes (Méndez, 2013).

En otras investigaciones se dice que las giberelinas son hormonas vegetales que regulan muchos de los procesos relacionados con el crecimiento y desarrollo de las plantas, como germinación, brotación, elongación del tallo, expansión foliar, desarrollo floral, entre otras (Sun, 2010). Es por esto que el objetivo del presente estudio es el de determinar los efectos de la aplicación de giberelinas en papas de la variedad canchán-INIA en la brotación de este tubérculo, en la ciudad de Chachapoyas (Departamento de Amazonas).

\section{MATERIAL Y MÉTODOS}

La presente investigación se realizó en los ambientes del laboratorio de Fitopatología y Entomología de la Universidad Nacional Toribio Rodríguez de Mendoza
deAmazonas (Figura 1).

Para obtener los tubérculos-semillas se siguió la metodología de selección positiva de semillas de papa mencionada por Obregón (2012). Dichas semillas fueron traídas del distrito de La Jalca (al sur de la provincia de Chachapoyas), donde se hizo una selección en un campo de cultivo que tenía 40 días de sembrado. En esta se evaluaron, escogieron y marcaron las plantas de papa que estuvieron completamente sanas, robustas, vigorosas y con buenos tallos. Posteriormente fueron monitoreadas, cosechadas y seleccionadas, obteniendo tubérculos sanos, uniformes, con un peso promedio de 40 a $60 \mathrm{~g}$ para luego ser trasladados al Laboratorio de Investigación en Fisiología y Biotecnología Vegetal de la Universidad Nacional Toribio Rodríguez de Mendoza.

Una vez en el laboratorio se realizó una limpieza profunda sumergiendo los tubérculos en un recipiente con agua, y restregándolos cuidadosamente con un cepillo hasta dejarlos libres de impurezas procedentes de la cosecha (Galarza, 2010). Luego fueron desinfectados con vitavax (Carboxín + Captán). Para la evaluación de parámetros como el porcentaje de brotación, número de brotes por tubérculo, longitud de brotes por tubérculo, y diámetro de brotes por tubérculo, se traba-
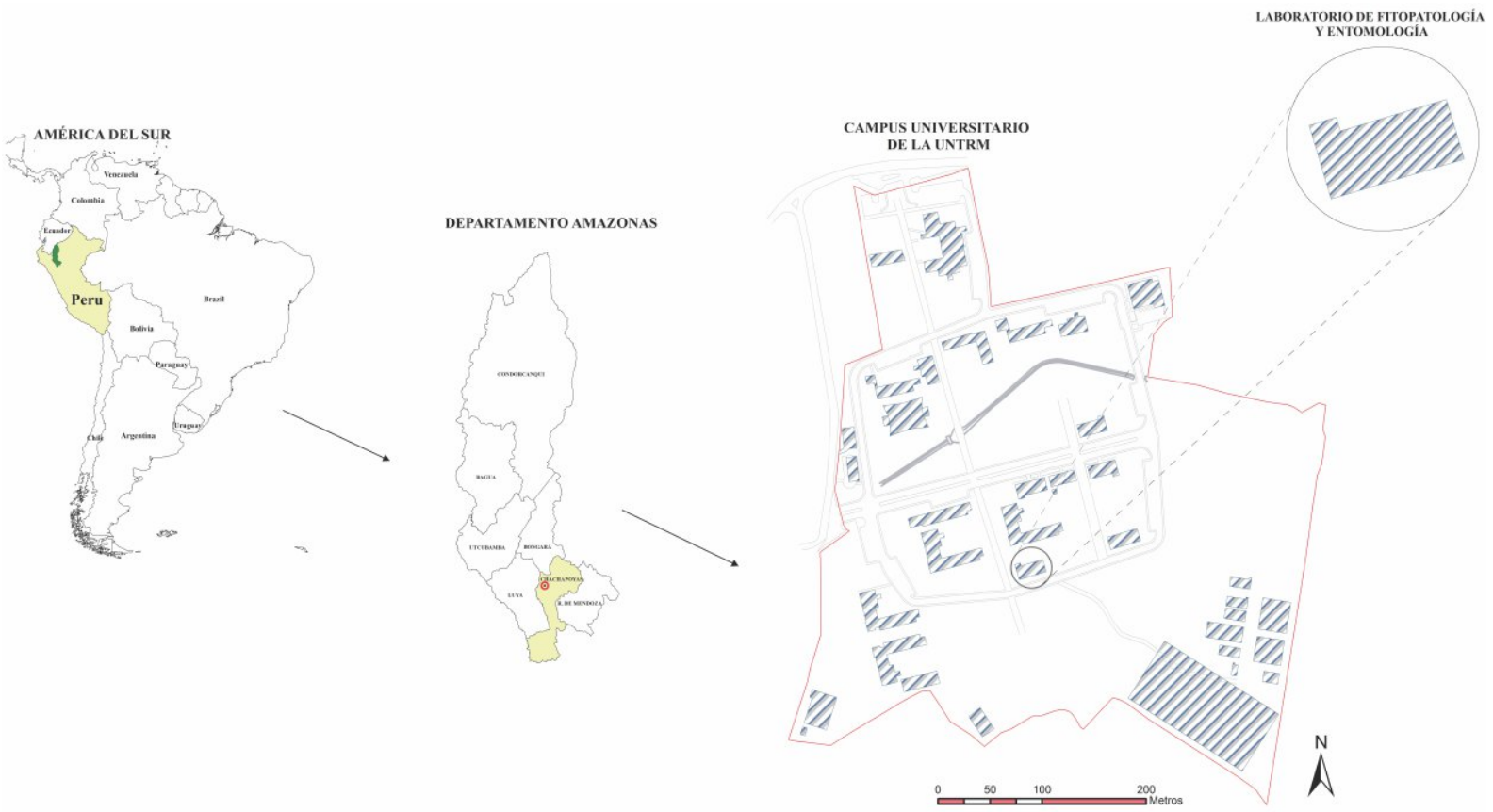

Figura 1. Mapa de ubicación de la zona de estudio en la ciudad de Chachapoyas (Departamento Amazonas). 
jó con un diseño completamente aleatorizado con seis tratamientos y tres repeticiones, incluido un testigo absoluto (Tabla 1).

Tabla 1. Tratamientos con giberelinas en estudio sobre los tubérculos

\begin{tabular}{cl}
\hline Tratamiento & \multicolumn{1}{c}{ Descripción } \\
\hline T1 (Testigo) & $\begin{array}{l}\text { Sin producto } \\
2,5^{*} \text { ml de Ryzup durante } 5 \\
\text { minutos de inmersión }\end{array}$ \\
T2 & $2,5^{*}$ ml de Ryzup durante \\
& 15 minutos de inmersión \\
T3 & $5^{*}$ ml de Ryzup durante 5 \\
& minutos de inmersión \\
T4 & $5^{*}$ ml de Ryzup durante 15 \\
& minutos de inmersión \\
T5 & $7,5^{*}$ ml de Ryzup durante 5 \\
& minutos de inmersión \\
T6 & $7,5 *$ ml de Ryzup durante \\
& 15 minutos de inmersión \\
\hline
\end{tabular}

* Dosis para 20 litros de agua

El proceso de instalación se realizó la primera semana de julio, y se inició con la distribución de siete unidades experimentales (javas de huevo). Posteriormente, se prepararon las dosis de acuerdo a las proporciones especificadas en la Tabla 1. Tres días después de la cosecha se procedió a la inmersión de los tubérculos en los diferentes tiempos y dosis de ácido giberélico, distribuyendo 30 tubérculos en cada java de huevos, las mismas que evitaron la acumulación de humedad y permitieron la libre circulación de aire, y en las cuales se llevaron a cabo los diferentes cambios fisiológicos en el tubérculo-semilla. Como una medida de protección contra el ataque de plagas se dejó una semana a luz difusa, para que la papa sufra un proceso de verdeamiento tornándose un poco amarga y menos apetitoso para los insectos (Figura 2). Seguidamente se cubrió con una manta negra durante una semana, para simular un ambiente cerrado que ayudó a la rápida aparición de brotes y el rompimiento de la dormancia. Siguiendo las consideraciones de diferentes autores dentro de ellos Salimi (2010) nos menciona que una semilla de papa pierde el periodo de reposo o dormancia cuando el $80 \%$ de los tubérculos tienen al menos un brote de por lo menos $2 \mathrm{~mm}$ de longitud, momento en el cual se considera una semilla brotada. Para ello se tomaron registros con intervalos de cuatro días hasta que todos los tubérculos completen el proceso de brotado. 39 días después de la cosecha y 33 días después de iniciado los tratamientos se vieron el inicio del brotamiento múltiple, periodo ideal para sembrar los tubérculos semilla.

En esta etapa se evaluó el porcentaje de la brotación, número de brotes por tubérculo, longitud de brotes por tubérculo y diámetro de brotes por tubérculo. Estos

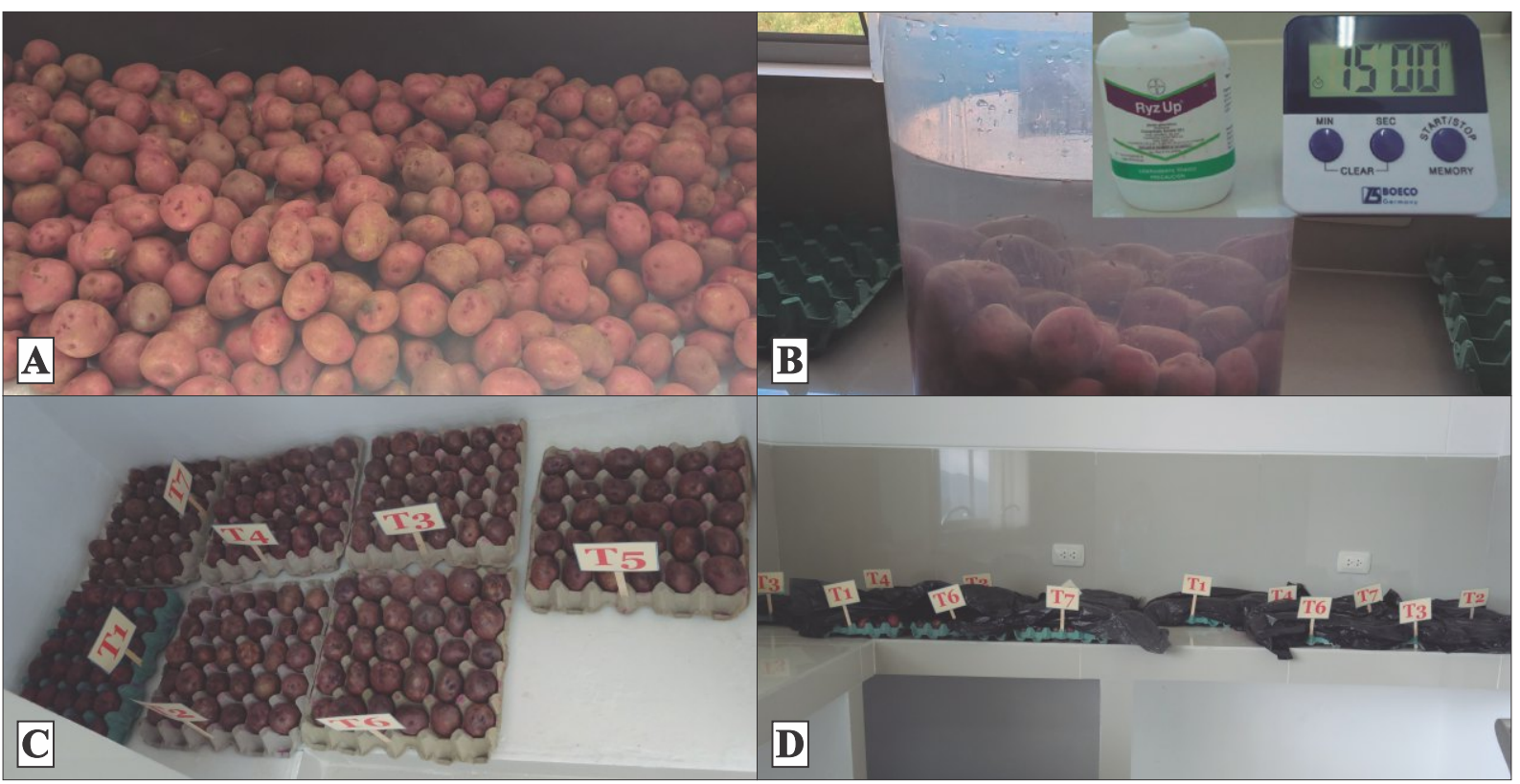

Figura 2. Limpieza de tubérculos (A), aplicación de Ag3 (B), semillas en luz difusa (C), simulación de ambiente oscuro (D). 
datos fueron tomados con la ayuda de un vernier digital sobre los tubérculos muestrales. La sistematización y análisis de los resultados obtenidos se efectuó con el programa Microsoft Excel, mientras que para el análisis de varianzas y las comparaciones múltiples se usó el software libre R x 64 versión 3.3.1.

\section{RESULTADOS}

\section{Porcentaje de la brotación (\%)}

El análisis de varianza mostró que existen diferencias estadísticas altamente significativas entre tratamientos, por lo cual se realizaron las comparaciones múltiples de medias según Duncan (Figura 3)

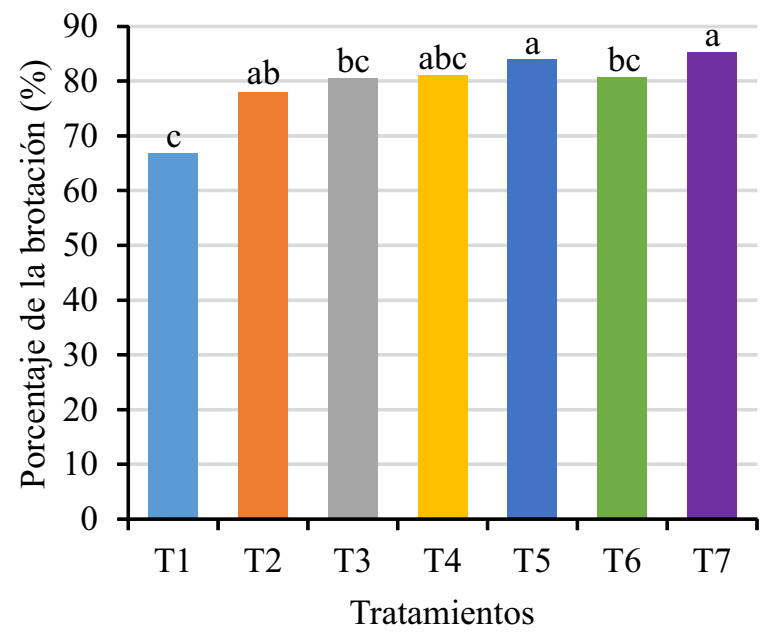

Figura 3. Porcentaje de la brotación de tubérculos.

Interpretando la prueba de Duncan en la Figura 3, se aprecia que el tratamiento $\mathrm{T} 7$ (7,5ml de Ryzup durante 15 minutos de inmersión) mostró el mayor valor en comparación con los demás tratamientos, más aún si se compara con el testigo (T1) que exhibe el menor valor en cuanto a esta variable.

\section{Número de brotes por tubérculo}

El análisis de varianza mostró que existen diferencias estadísticas altamente significativas entre tratamientos, por lo cual fue necesario realizar las comparaciones múltiples de medias según Duncan (Figura 4)

Interpretando la prueba de Duncan, en la figura 4, se aprecia que el tratamiento T5 ( $5 \mathrm{ml}$ de Ryzup durante 15 minutos de inmersión), mostró uno de los valores más altos ubicándose en el primer grupo $\mathrm{A}$, en comparación con los tratamientos T7, T6, T4 y T3, que se

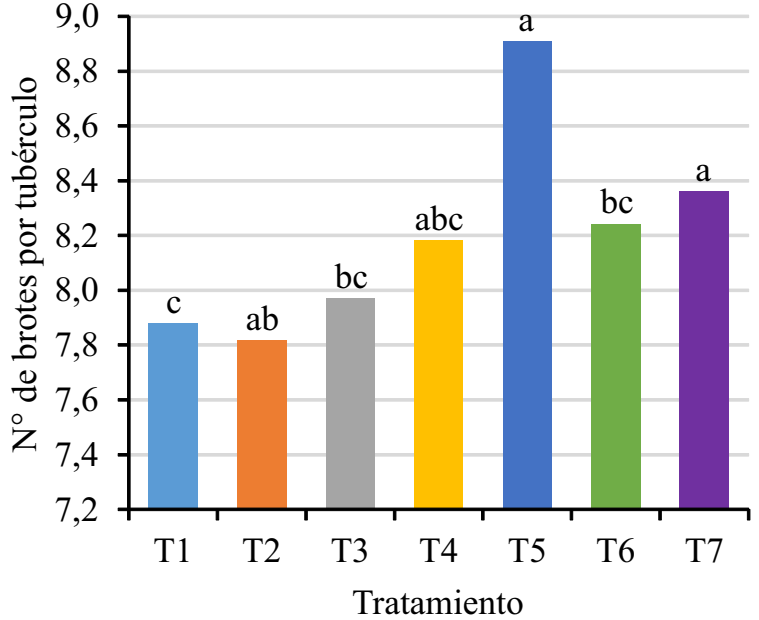

Figura 4. Número de brotes por tubérculo.

ubicaron en el segundo grupo AB. Cabe destacar que este valor es mayor si se compara con los tratamientos T1 y T2, que exhiben los valores más bajos en cuanto al número de brotes por tubérculo.

\section{Longitud de brotes por tubérculo (cm)}

Con respecto a la variable evaluada, longitud de brotes por tubérculo $(\mathrm{cm})$, el análisis de varianza mostró que existen diferencias estadísticas altamente significativas entre tratamientos, por lo cual se realizó la prueba de comparaciones múltiples de medias según Duncan (Figura 5)

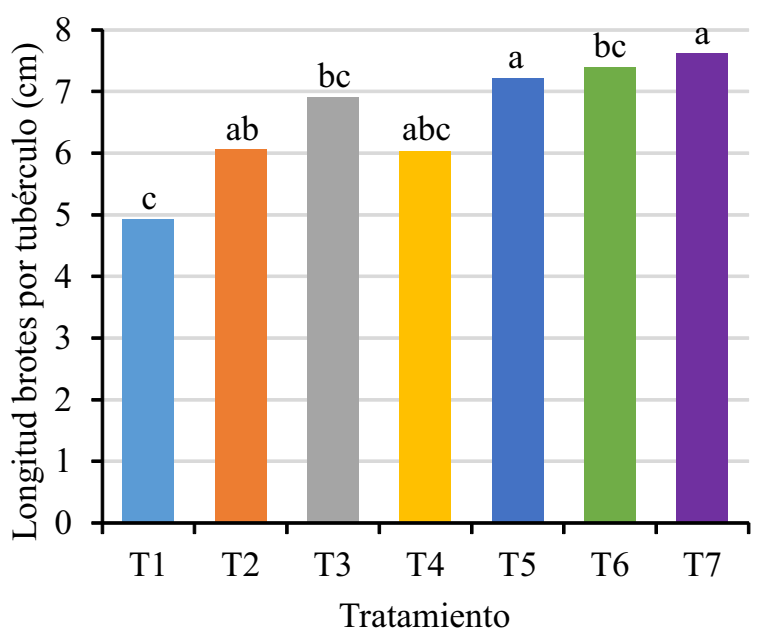

Figura 5. Longitud de brotes por tubérculo.

Interpretando la prueba de Duncan, en la figura 5, se aprecia que los tratamientos T7, T6, y T5 (con una dosis de 5 y 7,5 ml Ryzup sumergido en tiempos de 5 y 15 minutos) ocuparon los mayores valores ubicándose en el primer grupo A, en comparación con el T3 $(2,5 \mathrm{ml}$ 
de Ryzup durante 15 minutos de inmersión) que se ubicó en el segundo grupo AB. Por otra parte los tratamientos T2 y T6 ocuparon el tercer grupo B. Todos ellos obtuvieron valores más altos que el T1.

\section{Diámetro de brotes por tubérculo $(\mathrm{cm})$}

Con respecto a la variable evaluada, diámetro de brotes por tubérculo $(\mathrm{cm})$, el análisis de varianza mostró que existen diferencias estadísticas altamente significativas entre tratamientos, por lo cual se realizó la prueba de comparaciones múltiples de medias según Duncan (Figura 6).

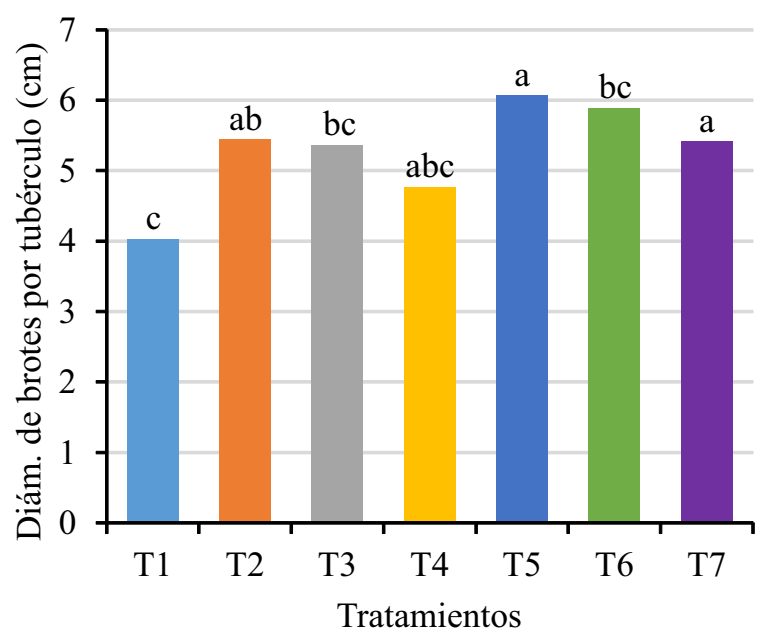

Figura 6. Diámetro de brotes por tubérculo

Interpretando la prueba de Duncan en la figura 6, se puede apreciar que existen 4 grupos de los cuales los que exhiben valores más altos son los tratamientos del grupo A, T5 y T6 (Ryzup a una dosis de 5 y 7,5ml sumergidos a un tiempo de 15 y 5 minutos respectivamente). Estos datos obtenidos están por encima de los demás tratamientos, más aún si se compra con el Testigo que exhibe uno de los valores más bajos.

\section{DISCUSIÓN}

En primer lugar, el periodo de reposo de los tubérculos de papa puede romperse o acelerarse por tratamientos físicos y fitohormonales, es así que las aplicaciones de giberelinas, citoquininas y auxinas rompen el reposo de los tubérculos (Vejarano y Morales, 2014). Así se muestran los resultados obtenidos en la presente investigación, siendo los tubérculos con aplicaciones de ácido giberélico los que rompieron el periodo de reposo en menor tiempo. Asimismo, se ha demostrado que los sistemas agrícolas tienen una influencia en los parámetros de calidad de los tubérculos, tales como las concentraciones de almidón y materia seca (Roinila et $a l ., 2003)$. Esto se puede demostrar en esta investigación, en la cual 33 días después de iniciadas las aplicaciones de giberilinas se logró un 85,26\% de brotación con el tratamiento $\mathrm{T} 7$, que estuvo compuesto por una dosis de 7,5ml de Ryzup sumergidos durante 15 minutos; similares resultados obtuvo Galarza (2010), quien menciona que los mejores resultados los obtuvo con 8 gr de New Gibb 10\%, sumergido durante 15 minutos. Estos datos obtenidos difieren mucho de la recomendación especificada en la ficha técnica del producto Ryzup, en la cual se recomienda que la dosis ideal para lograr la brotación inmediata sea de $2,5 \mathrm{ml}$ sumergido durante 15 minutos.

Con respecto al número de brotes por tubérculo no existe una relación directa con el porcentaje de brotación, por consiguiente el que más resaltó fue el T5 (5ml de Ryzup durante 15 minutos de inmersión), tratamiento que tuvo dosis y tiempo muy diferente al tratamiento T7. Cabe mencionar que en Dragicévic et al. (2008) resaltaron evidencias similares en su estudio sobre el tallo de la papa y la inducción al crecimiento del mismo a través de giberelinas. Las GA3, aumentan la aparición acelerada de numerosos brotes por tubérculo semilla tal como se observa en nuestros resultados de la figura 4 donde los tratamientos que contienen GA3 son superiores al testigo o tratamiento control. Estos resultados obtenidos comparados con un trabajo de investigación realizado por Salimi (2010), tienen coincidencia en cuanto a la reducción del tiempo y la aparición de muchos brotes de tubérculos en reposo que fueron tratados con GA3.

En cuanto a la longitud de brotes por tubérculo los resultados nos muestran un efecto significativo, el cual refleja la efectividad del GA3. Similares resultados obtuvo de nuevo Salimi (2010), quien trató microtubérculos de papa con ácido giberélico y disulfuro de carbono, logrando determinar que la longitud de los brotes después del tratamiento con CS2 y GA3 fue 
significativamente más alta que la de los controles o testigos, además que los microtubérculos tratados con GA3 mostraron una longitud de brotes significativamente suprior que los tratados con CS2.

Por su parte Vejarano y Morales (2014), evaluaron variables como longitud de brotes y diámetro de brotes por tubérculos, y encontraron que tubérculos semillas de la variedad canchán, 90 días después del almacenamiento, mostraron brotes apicales de $32 \mathrm{~mm}$. Estos resultados son comparables con los presentados en los tratamientos T5, T6 y T7 del presente estudio, donde se aprecia claramente la importancia de las aplicaciones de ácido giberélico (AG), que incrementan la síntesis de ARN y ADN (Rodríguez y Moreno, 2010). Estos incrementos preceden a la división celular, lo cual permite terminar el periodo de dominancia apical, e iniciar el periodo de brotamiento múltiple, es decir, comienzan a crecer las yemas inferiores y las yemas apicales, mostrándose el periodo de brotamiento múltiple como el ideal para sembrar los tubérculos semilla (Yang et al., 2010).

Autores como Alexopoulos et al. (2007) y Salimi (2010), determinaron que los brotes de los tubérculos tratados con GA3 tienden a ser largos, delgados, frágiles, y propensos a la rotura durante el manejo y la siembra. Esto puede limitar el uso comercial de GA3. Sin embargo, esto difiere de nuestros resultados, lo que puede estar relacionado a las horas de luz difusa que fue sometida cuando se dio la aparición de los primeros brotes.

\section{CONCLUSIONES}

El tratamiento $\mathrm{T} 7$ mostró excelentes resultados, ya que uniformizó el porcentaje de la brotación hasta un $85,26 \%$. En las unidades experimentales que estuvo bajo la influencia de este tratamiento se obtuvieron 8,36 brotes en promedio por tubérculo que estaban sanos y fuertes, es decir, con características físicas deseables, alcanzando a los 33 días de implantado el ensayo una longitud de $7,61 \mathrm{~cm}$. Con estos resultados se puede concluir que el tratamiento logró una eficaz ruptura de la latencia y estimulación de la brotación en los tubérculos de la papa (Solanum tuberosum) variedad canchán-INIA, y lo más importante, que es útil y accesible al agricultor ya que se traduce en una ganancia de tiempo de hasta dos meses, lo que posibilita la realización de hasta tres ciclos del cultivo al año.

\section{REFERENCIAS BIBLIOGRÁFICAS}

Alexopoulos, A., A. Konstantinos, S. Vemmos, y H. Passam. 2007. "The effect of postharvest application of gibberellic acid and benzyl adenine on the duration of dormancy of potatoes produced by plants grown from TPS”. Postharvest biology and technology 1: 54-62.

Birch P. R. J., G. Bryan, B. Fenton, E. Gilroy, I. Hein, J. T. Jones, A. Prashar, M. A. Taylor, L. Torrance, y I. K. Toth. 2012. "Crops that feed the world. Potato: are the trends of increased global production sustainable?" Food Security 4: 477-508.

Brush, S, R. Kesseli, R. Ortega, P. Cisneros, K. Zimmerer, y C. Quirós. 1995. "Potato diversity in the Andean center of crop domestication”. Conserv Biol 9: 1189-1198.

Cerna, J. 2011. El cultivo de la papa y su importancia. Lima (Perú): Agroindustria Definiciones y Realidades. Recuperado de: ht t p://inginieriaagroindustrialunt.blogspot.pe/2011/10/el-cultivo-de-lapapa-y-su-importancia.html

Deaquiz, Y. A., y Y.E . Burgos. 2015. "Efecto de la aplicación de giberelinas (GA3) sobre germinación de semillas de tomate (Solanum Lycopersicum L.) variedad Santa Cruz". Conexión AgropecuariaJDC 3:29-36.

Dragićević, I., R. Konjević, B. Vinterhalter, D. Vinterhalter, y M. Nešković. 2008. "The effects of IAA and tetcyclacis on tuberization in potato (Solanum tuberosum L.) shoot cultures in vitro". Plant Growth Regulation 54: 189-193.

Fernie, A. R., y L. Willmitzer. 2001. "Molecular and biochemical triggers of potato tuber development". Plant Physiol 127: 1459-1465. 
Galarza, P. 2010. Evaluación a la aplicación de giberelina (New gibb 10\%), para inducir a la brotación en tubérculos de papa (Solanum tuberosum). Tesis de Grado. Universidad Técnica de Ambato. Cavallos (Ecuador).

Hancock, R. D., W. L. Morris, L. J. Ducreux, J. A. Morris, M. Usman, S. R. J. Verrall, C. G. Fuller, R. Simpson, P. E. Zhang, E. Hedley, y M. A. Taylor. 2014. "Physiological, biochemical and molecular responses of the potato (Solanum tuberosum L.) plant to moderately elevated temperature". Plant, cell \& environment 37: 439-450.

INIA (Instituto Nacional de Innovación Agraria). 2012. Papa INIA 303 - Canchán. Lima (Perú): INIA Recuperado de: ht t p : / / w w w inia.gob.pe/prod$\begin{array}{lllll}\mathrm{s} & \mathrm{e} & \mathrm{r} & \mathrm{V} & \mathrm{i}-\mathrm{C}\end{array}$ cios/publicaciones/publicacion/tripticos/item /199-02-2012-papa-inia-303-canchan

Méndez, A. 2013 Evaluación de tres niveles de fertilización nitrogenada y su interacción con tres dosis de giberelinas en Aster (Aster sp). Tesis de Grado. Universidad Central del Ecuador. Quito (Ecuador). Recuperado de: http://www.dspace.uce.edu.ec/bitstream/250 00/2032/1/T-UCE-0004-34.pdf

MINAGRI (Ministerio de Agricultura y Riego). Perú lidera producción de papa en Latinoamérica. Lima (Perú): MINAGRI. Recuperado de: http://www.agraria.pe/noticias/peru-lideraproduccion-de-papa-en-latinoamerica-8337

Levy, D., y R. E. Veilleux. 2007. “Adaptation of potato to high temperatures and salinity - a review". American Journal of Potato Research 84: 487-506

Obregón, C. 2012. Producción de semilla de papa por selección positiva. Lima (Perú): ADERS Perú. Recuperado de: http://www.adersperu.com/propapa-per-cumple-10-aosinnovando

Peña, L. 2015. Fisiología y manejo de tubérculos semi- lla de papa. Lima (Perú): Redepapa. Recupe$\mathrm{r}$ a d o d e : https://medium.com/@redepapa/fisiologia-ymanejo-de-tuberculos-semilla-de-papab84693603380\#.ld7zq6kit

Reust, W. 1986. "Physiological age of the potato". Potato Res 29: 268-271.

Rodríguez, L. E., y L .P. Moreno. 2010. "Factores y mecanismos relacionados con la dormancia en tubérculos de papa. Una revisión". Agronomía Colombiana 28: 189-197.

Roinila, P., J. Väisänen, A. Granstedt, y S. Kunttu. 2003. "Effects of different organicfertilization practices and mineral fertilization on potato quality”. Biol. Agric.Hortic. 21: 165-194.

Salimi, K. 2010. "Effects of gibberellic acid and carbon disulphide on sprouting of potato minitubers". Scientia horticulturae 124: 14-18.

Senés-Guerrero, C., G. Torres-Cortés, S. Pfeiffer, M. Rojas, y A. Schüler. 2013. "Potato-associated arbuscular mycorrhizal fungal communities in the Peruvian Andes. Mycorrhiza” 24: 405-417.

Sun, T.P. 2010. "Gibberellin-GID1-DELLA: a pivotal regulatory module for plant growth and development". Plant Physiology 154: 567-570.

Torres, L., F. Montesdeoca, y J. Piedra. 2011. Manejo del tubérculo-semilla. Quito (Ecuador): CIPAgricultural research for development. Recu$\mathrm{p}$ e r a d o d e : http://cipotato.org/uncategorized/manejo-deltuberculo-semilla-de-la-papa/

Vejarano, A., y C. Morales. 2014. “Almacenamiento de la semilla de papa bajo condiciones de luz difusa". Pueblo continente 25: 3-101.

Vreugdenhil, D. 2007. "The canon of potato science: 39. Dormancy". Potato Research 50: 371-373.

Yang, W., X. Liu, J. Zhang, J. Feng, C. Li, y J. Chen. 2010. "Prediction and validation of conservative microRNAs of Solanum tuberosum L". Molecular biology reports 37: 3081-3087. 